\title{
Disparity in physician-patient communication by ethnicity: evidence from Bangladesh
}

\author{
Muhammad Zakaria ${ }^{1}$ B, Rezaul Karim, Murshida Rahman², Feng Cheng ${ }^{3}$ and Junfang $\mathrm{Xu}^{4^{*}}$
}

\begin{abstract}
Background: Physician-patient communication behavior (PPCB) is the primary process by which medical decisionmaking occurs and health outcome depends. Physician-patient communication differences may partly from the ethnic disparities. To examine this problem, this study aims to explore whether physician-patient communication differs by ethnicity during primary care medical consultations.
\end{abstract}

Methods: The study was conducted among the Bengali and ethnic minority patients $(N=850)$ who visited a physician for medical consultations. Data were collected using a structured post-consultation questionnaire. T-test was conducted to compare the communication between the Bengali and ethnic minority patients. Multiple linear regression analyses were performed to identify the factors associated with favorable communication behavior from the physicians.

Results: Bengali patients received more supportive communication behaviors from the Bengali doctors than that of ethnic minority patients including physicians' cheerful greetings, encouraging patients to express health problems and asking questions, listening carefully, responding to questions and concerns, explaining to patients about medical examination procedures, medication, probable side effects, discussing treatment options, involved the patients in decisions, and spending adequate time. Results of linear regression showed that respondents' level of education, internet use, knowledge about the health issue, having a pre-organized plan about the content of medical consultation, information seeking about the health problem, visiting female doctors, and a quiet ambience of the doctor's room are significantly associated with a better PPCB score for the Bengali patients. In contrast, age, being the resident of an urban area, perception of affecting a minor health problem, having a pre-organized plan about the content of medical consultation, patients' involvement in physicians' decision-making about the treatment, and talking time resulted in better physician-patient communication for the ethnic minority patients.

Conclusion: This study suggests that reducing disparity in the socio-economic status of the ethnic minority groups through development programs and educating healthcare providers on how to use patient-centered communication skills to engage with their patients is one solution to improve equity in the delivery of healthcare and ensure than patients are receiving high-quality treatment, no matter their race or ethnicity.

Keywords: Physician-patient communication behavior, Ethnic disparity, Bengali, Ethnic minority, Chittagong Hill tracts, Bangladesh

\footnotetext{
* Correspondence: junfangxuhappy1987@163.com

${ }^{4}$ Center for Health Policy Studies, School of Public Health, Zhejiang University

School of Medicine, Hangzhou 310058, China

Full list of author information is available at the end of the article
}

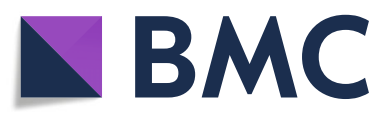

(c) The Author(s). 2021 Open Access This article is licensed under a Creative Commons Attribution 4.0 International License, which permits use, sharing, adaptation, distribution and reproduction in any medium or format, as long as you give appropriate credit to the original author(s) and the source, provide a link to the Creative Commons licence, and indicate if changes were made. The images or other third party material in this article are included in the article's Creative Commons licence, unless indicated otherwise in a credit line to the material. If material is not included in the article's Creative Commons licence and your intended use is not permitted by statutory regulation or exceeds the permitted use, you will need to obtain permission directly from the copyright holder. To view a copy of this licence, visit http://creativecommons.org/licenses/by/4.0/ The Creative Commons Public Domain Dedication waiver (http://creativecommons.org/publicdomain/zero/1.0/) applies to the data made available in this article, unless otherwise stated in a credit line to the data. 


\section{Introduction}

Physician-patient communication behavior (PPCB) is the primary process for health care service utilization. Research shows that successful PPCB can contribute to enhanced patient satisfaction and adherence to treatment and health outcomes, decreased costs of medical malpractice, and increased work satisfaction for physicians [1-4]. In addition, positive doctor-patient interactions could promote the sharing and obtaining of knowledge and overcome any differences of opinion during a physician's visit. However, creating a productive PPCB relationship is difficult when the doctor and the patient come from different racial or ethnic groups [1] because of various factors (i.e., language barriers and paradoxical attitudes about disease and illness). There is a growing body of empirical evidence showing that ethnic minority patients are received less patient-centered behavior from the discordant ethnic physicians and other health professionals, as cultural disparities between patients and physicians create trust barrier between the two parties, posing potential obstacles to the adequate provision of health services [3, 5-12]. Thus, failure to solve these problems may result in poor health outcomes for patients.

Racial or ethnic disparities in healthcare are defined as "differences in the quality of healthcare which are not due to access-related factors or clinic needs, preferences, and appropriateness of intervention" [13]. Ethnicity and culture influence the health outcomes through communicative actions of providers and patients in at least three ways [11]. Firstly, people of different ethnic backgrounds often speak different languages or dialects, even when the same language is generally spoken; there may still be cultural-specific uses of it, which contributes to communication related problems between physicians and patients. Secondly, preferred communication styles can vary across various cultural groups, particularly in regards to assertiveness and expressiveness. Finally, people of different ethnic backgrounds may have different methods of explanation regarding personal health and diseases and these, in turn, can affect the nature of medical consultations. These inequalities are exacerbated by prejudice about racism along with healthcare variables at the systemic level, such as language barriers, time constraints and regional disparities in quality of health treatment.

Previous studies have shown that cultural awareness, personal style, and language gaps often influence the outcome of clinical visits. For example, among a sample of general practice patients in the Netherlands, Harmsen et al. [14] examined the impact of cultural differences on mutual understanding and compliance, which found that interactions in consultations between general physicians and ethnic minority individuals were less effective than consultations with Dutch individuals. Some research [15,
16] indicated that contact with patients of various cultural backgrounds can also be troublesome, as doctors do not want to discuss cross-cultural issues. This could be for several reasons, including fear of sounding racist or prejudiced, a sense of inadequacy, a lack of cultural awareness, a fear of confusion or being rejected if suggestions are not culturally appropriate, and even ambiguity about whether or not the patient is an immigrant.

In Bangladesh, more than 45 ethnic minorities existed in the country prior to independence in 1971 [17]. Some cross-cultural or cross-ethnic studies in Bangladesh show that due to their low socio-economic status, minority groups often struggle to manage their livelihoods, which leads to deprivation of basic amenities, discrimination and health problems $[18,19]$. Although these minority communities have their own language, they are unable to use their language in every aspect of life due to the dominance or hegemony of Bengali language for its acceptance and inadequacy of their language. For example, people from ethnic minority groups may encounter language problems when admitted to a hospital or when visiting physicians. Even though the minority communities are gradually shifting to the Bengali language for survival in society, they may speak Bengali with a non-native accent which may lead to experiencing communicative difficulties and other ethnicity related problems when accessing health services.

Incorporating ethnic contexts into health communication research in health care is an important part of promoting health equity between the majority Bengali group and ethnic minority groups in Bangladesh [20, 21]. Tufnell et al. [22] observed that more than a third Bangladeshi population could not read or write in Bengali. This issue was more noteworthy among the older members in Bangladesh. It not only impedes communications with physicians but also means that these patients are unlikely to benefit from additional written medical materials. Moreover, Paternotte et al. [23] showed that language differences literally caused miscommunication in which it is not possible for the physician to achieve shared decisionmaking [23]. However, most of the related studies were conducted in western countries under primary care settings, including the USA and the UK [24], while very little study has been carried out in developing countries especially Bangladesh. The little existing studies in Bangladesh only focused on patients' satisfaction [25]; they did not examine the physician-patient relationship, communication between two parties, and its' link with cultural disparity. Under this background, this study aims to explore whether physician-patient communication differs by ethnicity in primary care medical consultations and then examine the factors influencing better communication behavior in primary care medical consultations in Bangladesh. The importance of this study lies in filling the 
research gap in this regard. Findings may also inform the public health practitioners about the existing communication patterns between physicians and patients by ethnic discordance that might serve as barriers of good compliance.

\section{Methods}

\section{Study design}

A hospital based cross-sectional survey was used to investigate the patients. This study was conducted in three district hospitals and three Upazila (sub-district) hospitals in the Chittagong Hill Tracts area (CHT). All the patients including Bengali and ethnic minority patients who visited physicians for primary care medical consultations in the study settings from August 19 to September 30, 2019 were incorporated to our study population.

Bangladesh is divided into 64 districts and the districts are further subdivided into 493 sub-districts or Upazila [26]. In each district, there is a district hospital which is responsible for delivering secondary and primary-care services to the whole district, whereas, at the Upazila level, there is an Upazila hospital or Upazila Health Complex (UHC) that provides all public-health programs, particularly primary healthcare services for the Upazila [27].

\section{Sample size and sampling procedures}

For this hospital based cross-sectional study, purposive sampling method was used to select the study areas, while study participants were selected using a systematic sampling method. Patients from three district hospitals and three Upazilas in three districts (Rangamati districts, Bandarban district, and Khagrachori district) under CHTs area were taken as participants. Finally, a total of 850 respondents were investigated and incorporated in the analysis. The proportion of participants [400 (47.1\%) were Bengali while 450 (52.9\%) were from three ethnic minority groups] in our study was in accordance with the overal population proportions of ethnicity in Bangladesh.

\section{Data collection}

Data were collected using a structured post-consultation questionnaire composed in the Bangla language. For pre-testing the questionnaire, 20 patients from the district hospital and 20 from an UHC were randomly selected. The questionnaire included the following four parts, a) patients' demographic and socio-economic characteristics; b) patients' cognitive, affective influences; c) physicians' predisposing and organizational context; d) physician-patient communication behavior (PPCB). PPCB was assessed using a scale that consists of 19 items developed by Wachira et al. [28], which consists of physicians' cheerful greetings, encouraging patients to explain their health problems and ask questions, giving information according to patients' needs, responding to questions and concerns, explaining to patients about medication, involving patients in decisions, and spending adequate time. To ensure the content was applicable to the context of Bangladesh, the scale was translated into the local language (i.e., Bangla) for appropriateness and convenience. Thereafter, the Bangla version was translated back to English to ensure consistency and accuracy of meaning. The translation of the questionnaire was performed by language experts in both cases. The appropriateness of the items of the scale was supported by adequate internal reliability $(\alpha=.76)$. Five-point Likert scale was used to measure each item related to physician-patient communication behavior as strongly disagree $=1$, disagree $=2$, neither agree nor disagree $=3$, agree $=4$, strongly agree $=5$.

The survey was guided and administered by six facilitators who were employed based on prior experience regarding data collection and eloquence in the local accent of the Bangla language for both Bengali and ethnic minority groups. Respondents were asked to respond to the questions at the end of their medical consultation. The informed written consent for participating in the study was obtained from each of the participants. Data collectors checked the filled questionnaire and asked the study participants to complete the questions if there was any missing information. Accordingly, there were no missing values in the study.

\section{Statistical analysis}

Cross-tabulation with chi-square $\left(\chi^{2}\right)$ analysis was used to examine the relationship between sociodemographic and other descriptive characteristics by respondents' ethnicity. Then, a two-tailed t-test was conducted to see the statistically significant differences between the Bengali and ethnic patients related to PPCB. Multiple linear regression analyses were also used to examine the factors influencing PPCB for the Bengali and ethnic minority groups. The score of 19 items regarding physician-patient communication behavior (PPCB) were computed and the overall PPCB score was the outcome variable. The distribution of the score of dependent variable was approximate normal distribution. Thus, the variables with a $p<.05$ in t-test and ANOVA were included in the linear regression models to determine the predictors of better PCCB. ANOVA values for better PPCB for the Bengali $(F=8.66, p<.001)$, and that for ethnic minority $(F=15.38, p<.001)$ showed that our multiple linear regression model performed well and would be a good predictor of the main outcome variable. Variables with $p<0.05$ were considered as statistically significant. 


\section{Results}

\section{Socio-demographic characteristics of participants by} ethnicity

Table 1 shows the socio-demographic characteristics of the study participants by their ethnic identity. Of them, $228(26.8 \%)$ had no formal education, while only 92 (10.8\%) had above 12 education years. Among the participants, $250(29.4 \%)$ were $>20-30$ years, whereas 168 (19.8) were $>40$ years old. Besides, 499 (58.7\%) were male and 351 (41.3\%) were female. For working industry, among the Bengali participants, $22 \%$ were involved in agriculture, $14.3 \%$ were in service, and $15.5 \%$ in business, while for the ethnic minority respondents, it was $29.3 \%$ (agriculture), 8.4\% (service) and 9.6\% (business) respectively. Among the Bengali participants, $19 \%$ had an income of BDT > 30,000 (good income), while only 4.7\% of ethnic minority participants had this amount of income.

\section{Patient and physician characteristics by ethnicity}

Physicians' predisposing, patients' cognitive, and organizational characteristics according to the ethnic identity is reported in Table 2. The Bengali participants (32.8\%) were more likely to be returning patients than their ethnic minority counterparts $(25.3 \%)$.

Table 1 Respondents' socio-economic characteristics by ethnicity $(N=850)$

\begin{tabular}{|c|c|c|c|c|c|}
\hline Variable $(N=850)$ & $n$ & Bengali (\%) & Ethnic Minority (\%) & $x^{2}$ & $p$ \\
\hline Gender & & & & 1.64 & .200 \\
\hline Female & 351 & $156(39.0)$ & $195(43.3)$ & & \\
\hline Male & 499 & $244(61.0)$ & $255(56.7)$ & & \\
\hline Age (years) & & & & 3.27 & .352 \\
\hline Up to 20 & 218 & $106(26.5)$ & $112(24.9)$ & & \\
\hline$>20-30$ & 250 & $114(28.5)$ & $136(30.2)$ & & \\
\hline$>30-40$ & 214 & $109(27.3)$ & $105(23.3)$ & & \\
\hline$>40$ & 168 & $71(17.8)$ & $97(21.6)$ & & \\
\hline Education & & & & 8.04 & .154 \\
\hline No education & 228 & $110(27.5)$ & $118(26.2)$ & & \\
\hline Up to class 5 & 113 & $53(13.3)$ & $60(13.3)$ & & \\
\hline class $>5-8$ & 131 & $69(17.3)$ & $62(13.8)$ & & \\
\hline class $>8-10$ & 163 & $70(17.5)$ & $93(20.7)$ & & \\
\hline Class $>10-12$ & 123 & $48(12.0)$ & $75(16.7)$ & & \\
\hline Class $>12$ & 92 & $50(12.5)$ & $42(9.3)$ & & \\
\hline Occupation & & & & 18.52 & .001 \\
\hline No job & 170 & $81(20.3)$ & $89(19.8)$ & & \\
\hline Agriculture & 220 & $88(22.0)$ & $132(29.3)$ & & \\
\hline Student & 260 & $112(28.0)$ & $148(32.9)$ & & \\
\hline Service & 95 & $57(14.3)$ & $38(8.4)$ & & \\
\hline Business & 105 & $62(15.5)$ & $43(9.6)$ & & \\
\hline Monthly household income (BDT) & & & & 85.33 & $<.001$ \\
\hline Up to 5000 & 165 & $35(8.8)$ & $130(28.9)$ & & \\
\hline$>5000-10,000$ & 228 & $110(27.5)$ & $118(26.2)$ & & \\
\hline$>10,001-20,000$ & 255 & $121(30.3)$ & $134(29.8)$ & & \\
\hline$>20,001-30,000$ & 105 & $58(14.5)$ & $47(10.4)$ & & \\
\hline$>30,000$ & 97 & $76(19.0)$ & $21(4.7)$ & & \\
\hline Internet use & & & & 0.05 & .814 \\
\hline Yes & 401 & $147(46.8)$ & $214(47.6)$ & & \\
\hline No & 449 & $213(53.3)$ & $236(52.4)$ & & \\
\hline
\end{tabular}

Columns against the categories of characteristics of each ethnic group sum to $100 \%$. Chi-square (x2) test was performed to depict the difference. BDT Bangladeshi Taka 
Table 2 Cognitive, organizational and other predisposing characteristics by the ethnicity

\begin{tabular}{|c|c|c|c|c|c|}
\hline Variable $(N=850)$ & $n$ & Bengali (\%) & Ethnic Minority (\%) & $x^{2}$ & $p$ \\
\hline Patient Type & & & & 5.68 & .017 \\
\hline Returning & 245 & $131(32.8)$ & $114(25.3)$ & & \\
\hline New & 605 & $269(67.3)$ & $336(74.7)$ & & \\
\hline Knowledge of the problem affected & & & & 27.34 & $<.001$ \\
\hline Poor & 625 & $263(65.8)$ & $362(80.4)$ & & \\
\hline Good & 225 & $137(34.3)$ & $88(19.5)$ & & \\
\hline Perception of the severity of the problem & & & & 4.87 & .301 \\
\hline Slight & 335 & $177(44.3)$ & $158(37.3)$ & & \\
\hline Moderate & 407 & $183(45.8)$ & $224(49.8)$ & & \\
\hline Extreme & 98 & $40(10.0)$ & $58(12.9)$ & & \\
\hline Waiting time (minutes) & & & & 1.41 & .495 \\
\hline$>0-20$ & 293 & $132(33.0)$ & $161(35.8)$ & & \\
\hline$>20-40$ & 224 & $103(25.8)$ & $121(26.9)$ & & \\
\hline$>40$ & 333 & $165(41.3)$ & $168(37.3)$ & & \\
\hline Gender of the doctor & & & & 4.52 & .034 \\
\hline Female & 228 & $121(30.3)$ & $107(23.8)$ & & \\
\hline Male & 622 & $279(69.8)$ & $343(76.2)$ & & \\
\hline The ambiance of the doctor's room & & & & 16.99 & $<.001$ \\
\hline Noisy & 459 & $218(54.5)$ & $241(53.6)$ & & \\
\hline Average & 278 & $111(27.8)$ & $167(37.1)$ & & \\
\hline Quiet & 113 & $71(17.8)$ & $42(9.3)$ & & \\
\hline Talking time (minutes) & & & & 54.11 & $<.001$ \\
\hline Up to 5 & 219 & $58(14.5)$ & $161(35.8)$ & & \\
\hline$>5-10$ & 197 & $180(45.0)$ & $17(38.9)$ & & \\
\hline$>10$ & 276 & $162(40.5)$ & $114(25.3)$ & & \\
\hline Patients' information seeking about the health problem & & & & 21.87 & $<.001$ \\
\hline Yes & 233 & $140(35.0)$ & $93(20.7)$ & & \\
\hline No & 617 & $260(65.0)$ & $357(79.3)$ & & \\
\hline Patients' involvement in decision-making of the treatment & & & & 6.96 & .008 \\
\hline Yes & 554 & $279(69.8)$ & $275(61.1)$ & & \\
\hline No & 296 & $121(30.2)$ & $175(38.9)$ & & \\
\hline
\end{tabular}

Columns against the categories of characteristics of each ethnic group sum to $100 \%$. Chi-square ( $x 2$ ) test was performed to depict the difference

In regards to the perception of health-related knowledge, the Bengali patients were more likely to have a good level of knowledge (30.8\%) compared to ethnic minority patients (18.4\%). In the case of the perception about the ambiance of the physicians' room, Bengali patients $(17.8 \%)$ were more likely to mention it as 'quiet' relative to their ethnic minority counterparts (9.3\%). Physicians had a higher likelihood to talk with the Bengali patients in comparison with ethnic minority patients. Moreover, ethnic minority patients were less likely to participate in medical consultations in contrast to Bengali patients $(p<.01)$.
Difference between the Bengali and ethnic minority patients regarding physicians' communication behavior Table 3 demonstrates that the Bengali patients reported better physician communication behavior than the ethnic minority patients did for 18 out of the 19 items for $\operatorname{PPCB}(p \leq .001)$, which included: physicians' greeting the patients, encouraging to express the health problems, listening carefully to the patients, understanding the patients, explaining the physical examination, explaining the lab tests needed, discussing treatment options with the patients, giving adequate information, and checking that the treatment plan(s) were acceptable to the 
Table 3 Mean score with standard deviation and independents samples t-test of different items relating to physician-patient communication behavior (PPCB) by ethnicity

\begin{tabular}{|c|c|c|c|c|}
\hline Items & Bengali & Ethnic Minority & $t$ & $p$ \\
\hline Your doctor greeted you in a way that made you feel comfortable & $3.53( \pm 1.16)$ & $3.27( \pm 1.09)$ & 3.44 & .001 \\
\hline Discussed your reason(s) for coming & $4.04( \pm) .74$ & $4.04( \pm .80)$ & .04 & .967 \\
\hline Encouraged you to express your thoughts concerning health problems & $3.99( \pm .76)$ & $3.48( \pm 1.01)$ & 8.41 & $<.001$ \\
\hline Listened carefully to you & $4.11( \pm .77)$ & $3.84( \pm .87)$ & 4.90 & $<.001$ \\
\hline Understood what you had to say & $3.93( \pm .94)$ & $3.62( \pm 1.04)$ & 4.56 & $<.001$ \\
\hline If a physical examination was required, the doctor fully explained & $3.19( \pm 1.23)$ & $2.47( \pm 1.24)$ & 8.43 & $<.001$ \\
\hline Explained the lab tests needed (e.g., blood, X-rays, ultrasound, etc.) & $3.22( \pm 1.15)$ & $2.45( \pm 1.26)$ & 9.31 & $<.001$ \\
\hline Discussed treatment options with you & $3.73( \pm 1.00)$ & $3.08( \pm 1.23)$ & 8.54 & $<.001$ \\
\hline Gave you as much information as you wanted & $3.72( \pm .92)$ & $3.20( \pm 1.01)$ & 7.77 & $<.001$ \\
\hline Checked to see if the treatment plan(s) was acceptable to you & $2.70( \pm 1.36)$ & $2.14( \pm 1.32)$ & 6.19 & $<.001$ \\
\hline Explained medications, if any, including possible side effects & $2.64( \pm 1.30)$ & $2.19( \pm 1.25)$ & 5.17 & $<.001$ \\
\hline Encouraged you to ask questions & $3.81( \pm .86)$ & $3.02( \pm 1.13)$ & 11.57 & $<.001$ \\
\hline Responded to your questions and concerns & $3.73( \pm 1.01)$ & $2.94( \pm 1.26)$ & 10.14 & $<.001$ \\
\hline Showed concern about you as a person & $3.97( \pm .82)$ & $3.76( \pm .94)$ & 3.61 & $<.001$ \\
\hline Involved you in decisions about your health as much as you wanted & $3.56( \pm .99)$ & $3.17( \pm 1.13)$ & 5.29 & $<.001$ \\
\hline Discussed next steps including any follow-up plans & $3.64( \pm 1.10)$ & $2.83( \pm 1.35)$ & 9.56 & $<.001$ \\
\hline Checked to be sure you understood & $3.63( \pm 1.09)$ & $2.83( \pm 1.27)$ & 9.84 & $<.001$ \\
\hline Spent the right amount of time with you & $3.98( \pm .76)$ & $3.43( \pm .95)$ & 9.34 & $<.001$ \\
\hline Overall, you were satisfied with your visit to the doctor today & $3.95( \pm .83)$ & $3.60( \pm 1.03)$ & 5.40 & $<.001$ \\
\hline
\end{tabular}

Table 4 Multiple linear regression analysis showing factors associated with physician-patient communication by the ethnicity

\begin{tabular}{|c|c|c|c|c|c|c|c|c|c|c|}
\hline \multirow[t]{2}{*}{ Variables } & \multicolumn{5}{|c|}{ Bengali } & \multicolumn{5}{|c|}{ Ethnic Minority } \\
\hline & B & SE & $\beta$ & $t$ & $p$ & B & SE & $\beta$ & $t$ & $p$ \\
\hline Constant & 48.36 & 2.93 & & 16.49 & $<.001$ & 27.811 & 3.73 & & 7.45 & $<.001$ \\
\hline Education of the respondents (Continuous variable) & .158 & .08 & .14 & 2.00 & .042 & .180 & .10 & .11 & 1.76 & .079 \\
\hline $\begin{array}{l}\text { Occupation of the respondents } \\
\text { (agriculture/no job vs. business/service/student) }\end{array}$ & .181 & .80 & .01 & .23 & .820 & .963 & 1.10 & .06 & .88 & .378 \\
\hline Age of the respondents (Continuous variable) & .048 & .03 & .10 & 1.65 & .101 & .120 & .03 & .20 & 3.61 & $<.001$ \\
\hline Area of Residence (rural vs. urban/sub-urban) & .022 & .63 & .00 & .03 & .972 & 1.670 & .79 & .09 & 2.13 & .034 \\
\hline $\begin{array}{l}\text { Marital status of the respondents } \\
\text { (married vs. single/widow/divorced) }\end{array}$ & 1.164 & .81 & .09 & 1.43 & .153 & 1.571 & 1.11 & .09 & 1.42 & .156 \\
\hline Respondents' Internet use (no vs. yes) & 1.937 & .80 & .16 & 2.42 & .016 & .970 & .94 & .06 & 1.03 & .303 \\
\hline Perception of the problem's severity (severe vs. minor) & -.285 & .57 & -.02 & -.50 & .616 & 1.724 & .71 & .10 & 2.42 & .016 \\
\hline Knowledge of health problem (poor vs. good) & 1.999 & .61 & .16 & 3.30 & .001 & -.708 & .88 & .03 & -.81 & .421 \\
\hline Plan about consultation before visit (no vs. yes) & 1.498 & .59 & .12 & 2.53 & .012 & 2.815 & .71 & .17 & 3.95 & $<.001$ \\
\hline Patients' information seeking (no vs. yes) & 2.136 & .58 & .17 & 3.68 & $<.001$ & .303 & .84 & .01 & .36 & .718 \\
\hline Involvement in decision-making (no vs. yes) & .642 & .61 & .05 & 1.05 & .292 & 3.231 & .70 & .19 & 4.60 & $<.001$ \\
\hline Gender of the doctors (male vs. female) & 1.322 & .63 & .10 & 2.11 & .035 & .228 & .80 & .01 & .28 & .777 \\
\hline Patient Status (new ns. returning) & .301 & .60 & .02 & .50 & .616 & .477 & .78 & .02 & .61 & .539 \\
\hline Talking time (Continuous variable) & .056 & .04 & .06 & 1.37 & .171 & .530 & .07 & .35 & 8.08 & $<.001$ \\
\hline \multirow[t]{2}{*}{ Ambience of the doctor's room (noisy vs. quiet) } & 1.762 & .81 & .11 & 2.19 & .029 & 1.944 & 1.15 & .07 & 1.69 & .091 \\
\hline & \multicolumn{2}{|c|}{$R^{2}=.25$} & \multicolumn{2}{|c|}{$F=8.66$} & $<.001$ & \multicolumn{2}{|l|}{$R^{2}=.35$} & \multicolumn{2}{|c|}{$F=15.38$} & $<.001$ \\
\hline
\end{tabular}


patients, among others. However, there was no significant difference by ethnicity in the mean score of the item whether physicians asked patients the reason(s) for coming $(t=.04, p=.967)$.

\section{Factors associated with PPCB by the ethnicity}

For Bengali patients, as Table 4 illustrates, respondents' level of education $(\beta=.14, p=.042)$, internet use $(\beta=.16, p=$ $.016)$, good knowledge about the health issue $(\beta=.16$, $p<.001$ ), having a pre-organized plan about the content of medical consultation $(\beta=.12, p=.012)$, patients' information seeking about the health problem $(\beta=.17, p<.001)$, female physicians $(\beta=.10, p=.035)$, and a quiet ambience of the physician's room $(\beta=.11, p=.029)$ are significantly associated with better PPCB. In contrast, for the ethnic minority patients, respondents' age $(\beta=.20, p<.001)$, being the resident of urban area $(\beta=.09, p=.034)$, perception of affecting a minor health problem $(\beta=.10, p=.016)$, having a preorganized plan about the content of medical consultation $(\beta=.17, \quad p<.001)$, patients' involvement in physicians' decision-making about the treatment $(\beta=.19, p<.001)$, and talking time $(\beta=.35, p<.001)$ are significantly associated with better PPCB.

\section{Discussion}

Our study found that concordant ethnicity between physician and patient was a key contributor for better patient-centered communication behavior, which illustrated the ethnic minority patients were less likely to receive better communication behavior from the discordant Bengali physicians. For example, the Bengali patients were more likely to receive friendly greetings from the physician that made them more comfortable with the medical consultations than that of the ethnic minority patients. Physician-patient communication begins through the form of a greeting. If not received warmly by the physician, the ethnic minority patient may feel discomfort, which likely will affect the entire consultation and lead them to communicate be less actively with the physician.

In terms of the affectionate behaviors, for example, encouraging patients to express their thoughts concerning health problems, listening carefully to and understanding what the patient says, our study observed that ethnic discordant physicians showed less concern towards ethnic minority patients. Similarly, we found that ethnic discrimination from Bengali physicians to the ethnic minority patients exists when it comes to discussing treatment options with the patients and providing adequate information. However, patient participation is an important element for effective physician-patient communication. Yet, patient satisfaction depends on the response behavior of the physician. We found a high inconsistency by ethnic discordance regarding encouraging the patients to ask questions and replying to the concerns. The general finding that Bengali physicians are less favorable towards the ethnic minority patients might be caused by the difference of language and culture, which is worthy of a future qualitative study $[11,12,28]$. This result was consistent with previous research conducted in Texas, USA that showed that physicians used less supportive talk or less patientcentered with non-white patients [11].

It is also reported that there was a vast discordance relating to the physicians' discussion on next steps, including any follow-up plans and checking to be sure the patient understood the content of the consultation. Spending adequate time with the patient is one of the most important predictors of patient satisfaction, which is linked to a greater adherence to therapy, return visit to the physician, and health improvements [29-33]. In our study, Bengali patients were more likely to receive sufficient time for medical consultation than that of the ethnic minority patients.

In terms of the patients' perception of being satisfied with the visit to the physician, the Bengali patients had a higher likelihood of expressing their satisfaction toward the physician than that of their ethnic minority counterparts. In Bangladesh, there still exists a strong cultural and traditional myth that health and diseases exist more among ethnic minority groups. Therefore, strategies should be carried out to address this serious misconception by physicians adopting better patient-centered behavior.

In addition, the favorable communication behavior from the physician was significantly higher among the patients who had a higher level of education and who used the Internet. This suggests that they may have the self-efficacy to engage in communicative behaviors with their clinicians thus influencing the communication dynamics and PPCB ratings [28]. Patients' age was also reported as an influencing factor for ethnic minority patients having better PPCB. It may be that older patients experience more severe diseases, which increases anxiety and increases the importance of communication style for the physician [34].

Patients' having a pre-organized plan about the content of consultation before visiting the physician was also reported as an essential predictor for both ethnic groups reporting better patient-centered communication behavior. Usually, the person who has a plan about the content of the consultation with the physician can talk and participate more appropriately, making the interaction more effective. Indeed, a statistical relationship was found in this study $(p<.001)$ between the preorganized plan of the consultation and patients' participation in decision making relating to the recommended treatment. 
The present study reported that female physicians were more likely to communicate more effectively with Bengali patients than male physicians. Our findings are in line with previous studies $[9,35,36]$, while Roter et al. [35] noted that female physicians prefer to use more patient-centered contact and promote more open and fair interaction than that of male physicians. Evidence has been found that female physicians tend to consider psychosocial issues through appropriate questioning and counseling, increased use of emotional and compassionate talk, and more constructive patient inputs. These elements were helpful for more effective patient-centered medical conversation [35].

It is worth to note that some variables were found to affect both Bengali patients and ethnic minority patients, such as a pre-organized plan about consultation before visit; while some variables only affected Bengali patients, such as level of education, internet use and gender of physician and some variables only affected ethnic minority patients, such as age, area of residence and appointment length. These differences may be explained by the discrepancy of socio-economic and cultural status between Bengali and ethnic minority patients, which is also worthy of a future qualitative study.

This study has some limitations. First, physicians' patient-centered behaviors were depicted based on selfreported information from the patients, which is subject to reporting errors. Second, considering there is a long line of patients waiting for a physician to be seen in medical centers due to the improper ratio between the number of physicians and patients; thus, physicians are always busy dealing with a large number of patients. Therefore, physicians are reluctant to complete any questionnaire about research. Moreover, quantitative measures of physician-patient interaction were examined rather than the quality of communication.

\section{Conclusion}

In conclusion, our research demonstrates that patients in concordant ethnic relationships report higher levels of physicians' patient-centered communication. Bengali patients received more supportive communication behaviors (e.g., physicians' cheerful greetings, affectionate behaviors, explaining the patients about a medical test, medication, and probable side effects, discussing the treatment options and involved the patients in decisions and spending adequate time) from the Bengali physicians than that of ethnic minority patients. Incorporating issues of cultural competency and social inequality into medical education and developing a culture-centered communication framework is an imperative aspect for reducing ethnic and socio-economic inequality. This will help ensure that all people can equally receive better health outcomes.

\section{Abbreviations}

ANOVA: Analysis of variance; BDT: Bangladeshi Taka; CHT: Chittagong Hill Tracts; PPCB: Physician-Patient Communication Behavior; UHC: Upazila Health Complex

\section{Acknowledgments}

The authors are grateful to the patients of the different district hospitals and Upazila health complexes who participated in this study, as well as the hospital/UHC authority for their permission and cooperation in collecting data.

\section{Authors' contributions}

All authors were responsible for the structure of this paper. MZ and JX designed the study and drafted the manuscript. JX contributed equally to this study and shares first authorship. RK, MR and FC contributed to the study's conception and design, interpretation of the data, draft manuscript and critical revisions of the paper. All authors approved the final versions for submission.

\section{Funding}

This work was partly supported by the Research and Publication Office of the University of Chittagong (no. 6474/2018), the National Natural Science Foundation of China (Project No. 71904171) and China Medical Board.

Availability of data and materials

All of the primary data has been included in the results. Additional materials with details may be obtained from the corresponding author.

\section{Ethics approval and consent to participate}

The study was conducted in accordance with the Declaration of Helsinki, and ethical approval for the study was provided by the Institutional Review Board for Human Subject Research, Research Centre for Public Health at Tsinghua University (THUSM/PHREC/2020400-015). Written informed consent was obtained from each participant before inclusion in the study.

Participation was voluntary, and the participants had an opportunity to reject or discontinue participation at any stage of the study.

\section{Consent for publication}

Not applicable.

\section{Competing interests}

All authors declare no competing interests.

\section{Author details}

${ }^{1}$ Department of Communication and Journalism, University of Chittagong, Chittagong 4331, Bangladesh. ²Department of English, Hamdard University Bangladesh, Munshiganj 1510, Bangladesh. ${ }^{3}$ Vanke School of Public Health, Tsinghua University, Beijing 100084, China. ${ }^{4}$ Center for Health Policy Studies, School of Public Health, Zhejiang University School of Medicine, Hangzhou 310058, China.

Received: 9 October 2020 Accepted: 9 February 2021

Published online: 24 February 2021

\section{References}

1. Ashton CM, Haidet P, Paterniti DA, Collins TC, Gordon HS, O'Malley K, et al. Racial and ethnic disparities in the use of health services: bias, preferences, or poor communication? J Gen Intern Med. 2003;18(2):146-52. https://doi. org/10.1046/j.1525-1497.2003.20532.x.

2. Brown JB, Stewart M, Ryan BL. Outcomes of patient-provider interaction. In: The Routledge handbook of health communication. London: Routledge; 2003. p. 155-76

3. Gupta N, Carr NT. Understanding the patient-physician interaction: potential for reducing health disparities. J Appl Soc Sci. 2008;2(2):54-65. https://doi. org/10.1177/193672440800200206.

4. Ong LM, De Haes JC, Hoos AM, Lammes FB. Doctor-patient communication: a review of the literature. Soc Sci Med. 1995;40(7):903-18. https://doi.org/1 0.1016/0277-9536(94)00155-m.

5. Ahmed R. Interpersonal health communication an ecological perspective. In: The handbook of global health communication, vol. 26; 2012. p. 144. https://doi.org/10.1002/9781118241868. 
6. Cooper LA, Roter DL, Johnson RL, Ford DE, Steinwachs DM, Powe NR. Patient-centered communication, ratings of care, and concordance of patient and physician race. Ann Intern Med. 2003;139(11):907-15. https:// doi.org/10.7326/0003-4819-139-11-200312020-00009.

7. Cooper-Patrick L, Gallo JJ, Gonzales JJ, Vu HT, Powe NR, Nelson C, Ford DE. Race, gender, and partnership in the patient-physician relationship. Jama. 1999;282(6):583-9. https://doi.org/10.1001/jama.282.6.583.

8. Doescher MP, Saver BG, Franks P, Fiscella K. Racial and ethnic disparities in perceptions of physician style and trust. Arch Fam Med. 2000;9:1156-63. https://doi.org/10.1001/archfami.9.10.1156.

9. Kaplan SH, Gandek B, Greenfield S, Rogers W, Ware JE. Patient and visit characteristics related to physicians' participatory decision-making style: results from the medical outcomes study. Med Care. 1995:1176-87 https:// www.jstor.org/stable/pdf/3766817.pdf.

10. Saha S, Komaromy M, Koepsell TD, Bindman AB. Patient-physician racial concordance and the perceived quality and use of health care. Arch Intern Med. 1999;159(9):997-1004. https://doi.org/10.1001/archinte.159.9.997.

11. Street RL Jr. Communication in medical encounters: an ecological perspective. In: Thompson TL, Dorsey AM, Miller Kl, Parrott R, editors. Handbook of health communication. Mahwah: Lawrence Erlbaum Associates; 2003. p. 63-89.

12. Matusitz J, Spear J. Effective doctor-patient communication: an updated examination. Soc Work Public Health. 2014;29(3):252-66. https://doi.org/10.1 080/19371918.2013.776416.

13. Smedley BD, Stith AY, Nelson AR. Institute of Medicine, committee on understanding and eliminating racial and ethnic disparities in health care. In: Unequal treatment: confronting racial and ethnic disparities in healthcare. Washington (DC): National Academies Press (US); 2003. https:// doi.org/10.17226/12875.

14. Harmsen $H$, Meevswesen $L$, Van Wieringen J, Bernsen R, Bruijnzeels M. When cultures meet in general practice: intercultural differences between GPs and parents of child patients. Patient Educ Counsel. 2003;51:99-106. https://doi.org/10.1016/s0738-3991(02)00195-7.

15. Berger JT. Culture and ethnicity in clinical care. Arch Intern Med. 1998; 158(19):2085-90. https://doi.org/10.1001/archinte.158.19.2085.

16. Lloyd M, Bor R. Communication skills for medicine. Edinburgh: Churchill Livingstone; 1996.

17. Barman DC, Neo MS. Human rights report 2013 on indigenous peoples in Bangladesh. Dhaka: Kapaeeng Foundation; 2014.

18. Uddin ME. Cross-cultural social stress among Muslim, Hindu,Santal and Oraon communities in Rasulpur of Bangladesh. Int J Sociol Soc Pol. 2011; 31(5/6):361-88. https://doi.org/10.1108/01443331111141309.

19. Uddin E. Ethnic disparity in family socio-economic status in Bangladesh: implication for family welfare policy practice. Glob Soc Welf. 2015;2(1):29-38. https://doi.org/10.1007/s40609-014-0021-x.

20. Masud Ahmed S. Differing health and health-seeking behaviour: ethnic minorities of the Chittagong Hill tracts, Bangladesh. Asia Pacific J Public Health. 2001;13(2):100-8. https://doi.org/10.1177/101053950101300208.

21. Saha, P. S., 2015. Indigenous people sill in Back (Adibashira ekhono pichiye). The Prothom-alo. https://www.prothomalo.com/bangladesh/article/597457

22. Tufnell DJ, Nutall K, Raistrick J, Jackson TL. Use of translated written material to communicate with non-English speaking patients. Br Med J. 1994;309: 992. https://doi.org/10.1136/bmj.309.6960.992.

23. Paternotte $E$, van Dulmen S, van der Lee N, Scherpbier AJ, Scheele F. Factors influencing intercultural doctor-patient communication: a realist review. Patient Educ Couns. 2014;98(4):420-45. https://doi.org/10.1016/j. pec.2014.11.018.

24. Ishikawa H, Takayama T, Yamazaki Y, Seki Y, Katsumata N. Physician-patient communication and patient satisfaction in Japanese cancer consultations. Soc Sci Med. 2002:55(2):301-11. https://doi.org/10.1016/s0277-9536(01)00173-3.

25. Andaleeb SS, Siddiqui N, Khandakar S. Patient satisfaction with health services in Bangladesh. Health Policy Plan. 2007;22(4):263-73. https://doi. org/10.1093/heapol/czm017.

26. Bangladesh Bureau of Statistics (BBS). Bangladesh statistics 2019. Dhaka: BBS; 2020. https://bbs.portal.gov.bd/site/page/23b9eb9e-160e-4407-a49f-5722 bc57f528/

27. Ministry of Health and Family Welfare (MOHFW). Health bulletin 2017. Directorate General of Health Services (DGHS): Dhaka; 2018. https://dghs. gov.bd/images/docs/Publicaations/HealthBulletin2017Final13_01_2018.pdf

28. Wachira J, Middlestadt S, Reece M, Peng CYJ, Braitstein P. Psychometric assessment of a physician-patient communication behaviors scale: the perspective of adult HIV patients in Kenya. AIDS Res Treatment. 2013;2013. https://doi.org/10.1155/2013/706191.

29. Cegala DJ, Post DM. The impact of patients' participation on physicians' patient centered communication. Patient Educ Couns. 2009;77:202-8. https://doi.org/10.1016/j.pec.2009.03.025.

30. Grayson-Sneed KA, Dwamena FC, Smith S, Laird-Fick HS, Freilich L, Smith RC. A questionnaire identifying four key components of patient satisfaction with physician communication. Patient Educ Couns. 2016;99(6):1054-61. https://doi.org/10.1016/j.pec.2016.01.002.

31. Levinson W, Lesser CS, Epstein RM. Developing physician communication skills for patient-centered care. Health Aff. 2010;29(7):1310-8. https://doi. org/10.1377/hlthaff.2009.0450.

32. Marcum ZA, Sevick MA, Handler SM. Medication nonadherence: a diagnosable and treatable medical condition. JAMA. 2013;309(20):2105-6. https://doi.org/10.1001/jama.2013.4638.

33. Zolnierek KBH, DiMatteo MR. Physician communication and patient adherence to treatment: a meta-analysis. Med Care. 2009;47(8):826. https:// doi.org/10.1097/MLR.0b013e31819a5acc.

34. Buller MK, Buller DB. Physicians' communication style and patient satisfaction. J Health Soc Behav. 1987:375-88. https://doi.org/10.2307/213 6791.

35. Roter DL, Hall JA, Aoki Y. Physician gender effects in medical communication: a meta-analytic review. JAMA. 2002;288(6):756-64. https:// doi.org/10.1001/jama.288.6.756.

36. Weisman CS, Teitelbaum MA. Women and health care communication. Patient Educ Couns. 1989;13(2):183-99. https://doi.org/10.1016/0738-3991 (89)90060-8.

\section{Publisher's Note}

Springer Nature remains neutral with regard to jurisdictional claims in published maps and institutional affiliations.
Ready to submit your research? Choose BMC and benefit from:

- fast, convenient online submission

- thorough peer review by experienced researchers in your field

- rapid publication on acceptance

- support for research data, including large and complex data types

- gold Open Access which fosters wider collaboration and increased citations

- maximum visibility for your research: over $100 \mathrm{M}$ website views per year

At BMC, research is always in progress.

Learn more biomedcentral.com/submissions 